\title{
Uso de las tecnologías de la información en la educación superior
}

\section{Use of information technologies in higher education}

\author{
Abel Polivio Remache Coyago \\ Universidad Central del Ecuador, Ecuador \\ Edwin Giovanny Puente Moromenacho \\ Universidad Internacional del Ecuador, Ecuador \\ Gustavo Adolfo Moreno Jiménez \\ Universidad Internacional SEK, Ecuador
}

Autor para correspondencia: abelbelo@hotmail.com, epuente@internacional.edu.ec, gustavoadolfo90@gmail.com.

Fecha de recepción: 20 de Octubre - Fecha de aceptación: 20 de diciembre de 2016

\section{Resumen}

El presente documento muestra el surgimiento de los Sistemas de Gestión del Aprendizaje (LMS), y su introducción en la Educación Superior, más que nada se realiza un análisis de los parámetros fundamentales que se debe tener en cuenta en el manejo de una plataforma virtual dentro de una institución. Se analiza también los retos actuales de la educación superior respecto a la internacionalización de la educación, las ventajas y criterios al momento de elegir e introducir una LMS en un establecimiento, así como las medidas básicas necesarias a tener en cuenta respecto a la fiabilidad y eficacia de las TIC.

Palabras clave: Gestión del Aprendizaje; TIC; educación superior

\begin{abstract}
This document shows the emergence of Learning Management Systems (LMS), and its introduction in Higher Education, also an analysis of key parameters to be considered in the management of a virtual platform is made within of an institution. Current challenges of higher education regarding the internationalization of education, benefits and criteria when choosing and introducing a LMS in an establishment as well as the basic measures needed to consider regarding the reliability and efficiency is also analyzed of ICTs.
\end{abstract}

Key Words: Learning management; ICT; higher education 


\section{Introducción}

El inicio de las tecnologías de la información respecto a la educación superior, toma curso con la introducción de la formación académica por correspondencia, en donde se entregaba la información a través de papel, casetes, videocintas, discos de información, etc. El tiempo que tomaba el proceso del sistema de comunicación era muy lento y los resultados de aprendizaje eran muy cuestionados. Numerosos recursos tecnológicos que eran utilizados como medio de comunicación general fueron convertidos en medios de aprendizaje, tales como la televisión, las videoconferencias, redes telefónicas. Los inconvenientes siempre fueron la distancia y acompañamiento que un docente puede entregar a un alumno, esto comparado con una clase presencial en donde las relaciones de profesor alumno son directas y se maneja el sistema de comunicación de forma instantánea. El despliegue y mejoramiento del internet junto con sus herramientas tecnológicas han hecho que este espacio se reduzca drásticamente. Las videoconferencias, chats online, mails electrónicos, plataformas especializadas, repositorios, han fortalecido el sistema educativo a distancia o virtual.

Actualmente todas las instituciones de educación superior han admitido las TIC como medio de transformación y mejoramiento de la transferencia de conocimiento en su sistema educativo, y muchas instituciones a nivel internacional acogen estas tecnologías para la formación y entrega de títulos a nivel profesional. El reto actual se basa en que estas profesiones sean reconocidas y acreditadas en diferentes espacios (países, regiones u otras instituciones de educación superior), para esto se debe evaluar y demostrar que sus estudiantes también ocupan un lugar importante por el reconocimiento por sus logros alcanzados y conocimientos adquiridos.

Sancho, menciona varias instituciones educativas que en la actualidad ofertan carreras universitarias a distancia tales como: Athena Universtity, California Coast Universtity, International Universtity, Virtual Online Universtity, Campus Virtual del Instituto Tecnológico de Enseñanza Superior de Monterrey y la Universtity Oberta de Cataluña. (Sancho, 2002, pág. 17).

\subsection{Relación de las plataformas educativas y el docente}

De manera directa, el profesor debe convertirse en una guía que facilite el uso de los recursos tecnológicos, con el fin de que el alumno pueda acceder a ellos y sacarles provecho. Al hablar de aprovechamiento de los recursos a nivel superior, se puede decir que el fin está en transferir eficientemente el conocimiento para tener la capacidad de comprenderlo y crear nuevo conocimiento. Para lograr dichas características se deben asociar varias teorías actuales de aprendizaje de autores como Piaget, Vygotsky, Skinner, Brunner, etc.; además los docentes deben aprender y perfeccionar el uso y aprovechamiento de las tecnologías actuales, por lo que Salinas, sugiere lo siguiente:

$\checkmark$ Conocimiento y dominio del potencial de las tecnologías

$\checkmark$ Interacción con la comunidad educativa y social en relación a los desafíos que trae la sociedad del conocimiento.

$\checkmark$ Conciencia de las necesidades formativas de la sociedad

$\checkmark$ Capacidad de planificar el desarrollo de su carrera profesional. (Salinas, 2004, pág. 12) 
A lo que se añadiría como elemento indispensable:

$\checkmark$ Conocimiento y manejo de las teorías de aprendizaje aplicadas al entorno.

Poco a poco las tecnologías se han ido optimizando y acoplando, por lo que se han creado nuevos escenarios de aprendizaje con diversos resultados. Los sistemas de educación a distancia se han reforzado gracias a la creación de entornos de aprendizaje y plataformas de enseñanza virtual a todo nivel. El control virtual por medio de las plataformas es más eficiente ya que varias plataformas permiten la vigilancia (tareas, calificaciones, tiempo de trabajo, lecturas etc.) casi total de sus alumnos por este medio.

La distancia comunicativa entre el docente y el alumno se acorta por la intervención de dichos entornos virtuales conocidos también como LMS (Learning Management System). Hace pocos años estos entornos eran muy criticados, pero rápidamente se han ido corregido temas como: el papel de los docentes y su intervención en el aprendizaje, el acompañamiento a los alumnos, el tiempo de comunicación por medio de estos canales, la interacción entre una clase virtual y una clase real con sus resultados; además existen varias ventajas sobre una clase presencial, puesto que muchos sistemas permiten la reproducción de clases dictadas, acceso a presentaciones e interacción con otros alumnos y varias universidades interrelacionadas.

No se debe confundir varios nombres que representen un significado semejante en lo que se refiere a plataformas informáticas o TIC utilizadas en varias instituciones tal como plantea Salinas: en los últimos tiempos hemos oído hablar de 'enseñanza virtual', 'campus virtual' 'universidad virtual', y más recientemente 'e-learning', 'blended learning', etc., solamente en el mundo educativo, sin constituir otra innovación. (Salinas, 2004, pág. 17), a los que se pueden sumar los términos: e-learning, Distance Learning, Computer-Based Learning, WebBased Learning, Collaborative Learning, Virtual Learning, Mixed Learning, Blended Learning, Mediated Learning, Digital Education, Cybereducation, Online Education, Distance Education, etc.

La aparición de los LMS o Sistemas de Gestión de Aprendizaje se debe a la asociación de varias herramientas tecnológicas y su administración que van evolucionando de manera global acorde a las necesidades de sus usuarios y al objetivo principal de mejorar los sistemas educativos junto con la investigación. Dentro de los recursos actuales implementados a los sistemas de gestión de aprendizaje están las CBT (Computer-Based Training), WBT (Web-Based Training), y BBT (Bulletin Board Systems) como las más importantes.

La CBT (Computer-Based Training) junta en un solo contexto recursos como: textos, gráficos, animaciones, presentaciones, formatos de audio y video, las cuales deben estar interrelacionadas e interactivas. La WBT (Web-Based Training) además de poseer las utilidades de una CBT junta los recursos y herramientas que puede brindar el internet (a nivel mundial), por lo que es un sistema que depende del flujo de datos y de sus sistemas operativos. Los BBS (Bulletin Board Systems) los componen los mails y foros, aplicaciones de chat y programas de net-meeting hasta sistemas de tecnología compleja como el streaming del audio y vídeo, y la videoconferencia. (Schneckenberg, 2004, pág. 145) 


\section{La educación superior frente a la acreditación y las Tic}

Tomando en cuenta el ejemplo europeo, las instituciones de educación superior tratan en lo posible ser reconocidas internacionalmente tanto en educación a distancia, virtual y presencial a través de tratados (Proceso de Bolonia). Es por tal motivo que se crearon las ECTS (European Credit Transfer System) con la finalidad de facilitar el proceso de intercambio y movilidad estudiantil. El Espacio Europeo de Educación Superior define a las ECTS como un sistema que reconoce y mide el trabajo que deben realizar los estudiantes para la adquisición de los conocimientos, capacidades, y destrezas necesarias para superar las diferentes materias de su plan de estudios (EEES, 2015), estas deben estar alineadas a las necesidades de la sociedad y su contenido debe ser mejorado y actualizado continuamente.

De esta forma es imperativo el uso de TIC en la Educación Superior para solventar este proceso de convergencia. Analizando los datos obtenidos de la Revista de Educación (Paredes \& Estebanell, 2005), se realizó una encuesta a profesores, con calificaciones en rangos de 0 a 6 , en donde la puntuación 0 figura como nada importante y 6 es muy importante; los datos consultados para definir valores de importancia y reflejados en la Tabla 1 fueron los siguientes:

Tabla 1. Datos consultados a profesores

\begin{tabular}{ll}
\hline $\mathbf{1}$ & Procesos ECTS \\
\hline $\mathbf{2}$ & Preparación materiales multimedia \\
\hline $\mathbf{3}$ & Diseño de actividades de aprendizaje a distancia e interactivas \\
\hline $\mathbf{4}$ & Diseño de páginas web de apoyo a la docencia \\
\hline $\mathbf{5}$ & Creación de páginas web con contenidos didácticos \\
\hline $\mathbf{6}$ & Procedimientos de Organización y Gestión \\
\hline $\mathbf{7}$ & Adaptación de las materias al ECTS \\
\hline $\mathbf{8}$ & Nuevas metodologías didácticas \\
\hline $\mathbf{9}$ & Nuevos métodos de evaluación didáctica \\
\hline $\mathbf{1 0}$ & Utilización de la TIC en la Docencia \\
\hline $\mathbf{1 1}$ & Utilización de la TIC en la Investigación \\
\hline $\mathbf{1 2}$ & Aplicaciones Innovadoras de las TIC con repercusiones en la metodología docente \\
\hline $\mathbf{1 3}$ & Herramientas de comunicación para la tutoría \\
\hline $\mathbf{1 4}$ & Gestión de asignaturas a través de plataformas de enseñanza \\
\hline
\end{tabular}

En el gráfico 1, con los resultados obtenidos (Sin tomar en cuenta los valores entre 1 y 5) se evidencia que para los docentes el tema de reconocimiento de créditos, la aceptación de sus materias a las ECTS y el uso de nuevas metodologías didácticas son muy importantes. Esto refleja que a toda costa el reconocimiento de las materias a nivel internacional y la transferencia de conocimiento son de gran importancia para un profesor y para las instituciones de educación superior. Pero de manera contraria la capacitación de manejo e introducción de las TIC tiene una jerarquía menos relevante para un docente, lo que viene a ser una contradicción en la cual se debe focalizar los esfuerzos de mejora en la educación superior. (Paredes \& Estebanell, 2005, págs. 236-241) 


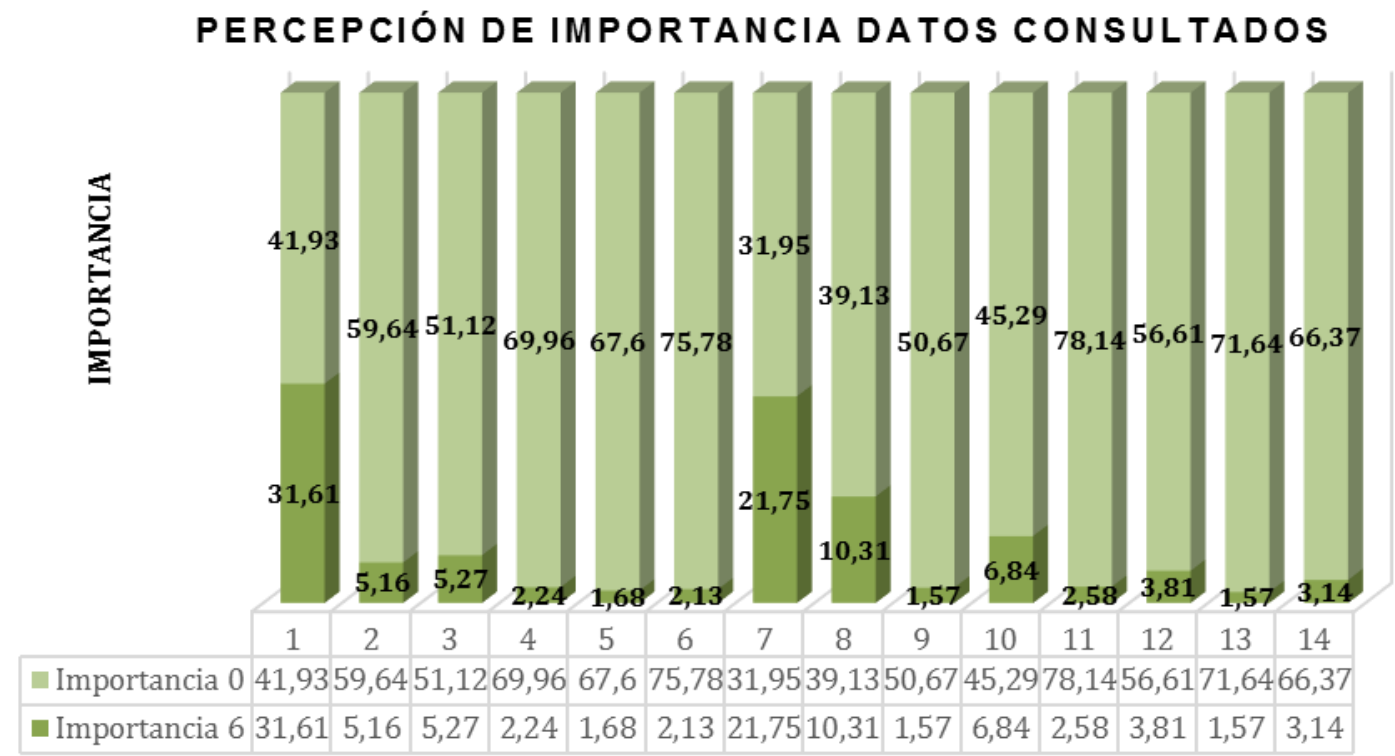

Gráfico 1. Percepción de importancia entre datos consultados

Se puede analizar también el estado de introducción de las TIC en las Instituciones de educación técnica superior, contrastando la información que proporciona el ranking académico mundial de universidades realizado por el Centro para la Clasificación Mundial de Universidades de la Universidad de Shanghai Jiao Tong, en donde se sitúa a las siguientes Universidades dentro de las mejores en el campo de las Ingenierías (en orden de clasificación): Massachusetts Institute of Technology (MIT), Stanford University, University of California-Berkeley, University of Illinois at Urbana-Champaign, The University of Texas at Austin, Georgia Institute of Technology, University of California, Santa Barbara, The Imperial College of Science, Technology and Medicine, University of Michigan-Ann Arbor y Carnegie Mellon University entre las 10 mejor puntuadas (Center for World-Class Universities of Shanghai Jiao Tong University, s.f.). De esta manera en la Tabla 2, se analiza las plataformas educativas utilizadas como medio de formación en las principales universidades que constan dentro del ranking mundial en ingenierías, en donde absolutamente todas estas instituciones dan suma importancia a las TIC, puesto que se evidencia que han encontrado ventajas en lo que se refiere a la mejora del sistema educativo, ya que pueden trabajar directamente con repositorios de textos, artículos indexados, libros, multimedia, etc., además los estudiantes pueden interconectarse de mejor manera con otras instituciones y diversos usuarios. Algunas plataformas educativas están diseñadas incluso para realizar investigaciones y contar con el aporte a nivel mundial de otras personas o instituciones. A continuación se puede observar las características de varias Universidades que se encuentran dentro del ranking mundial:

Tabla 2. Plataformas utilizadas en las mejores universidades a nivel de ingeniería 


\begin{tabular}{|c|c|c|}
\hline 1 & $\begin{array}{l}\text { MIT (Massachusets Institute Of } \\
\text { Technology) }\end{array}$ & $\begin{array}{l}\text { OCW (Open Course Ware)- Mitx Y Edx } \\
\text { MOOC (Massive Open Online Course) } \\
\text { Seguimiento Estudiantil Y Apertura Mundial }\end{array}$ \\
\hline 2 & Standford University & $\begin{array}{l}\text { Vice Provost For Online Learning (VPOL) } \\
\text { Stanford Openedx } \\
\text { Dedicada A Iniciativas De Enseñanza Online E Investigación }\end{array}$ \\
\hline 3 & $\begin{array}{l}\text { University Of California - } \\
\text { Berkeley }\end{array}$ & UC Berkeley Extension Online, UC Berkeley Ed X, Moocs \\
\hline 4 & $\begin{array}{l}\text { University Of Illinois At } \\
\text { Urbana-Champaign }\end{array}$ & $\begin{array}{l}\text { CITL Center For Innovation, Teaching And Learnig } \\
\text { Metodologías Basadas En La Investigación, Las Tecnologías } \\
\text { Educativas Innovadoras Y Prácticas De Evaluación Integrales }\end{array}$ \\
\hline 5 & $\begin{array}{l}\text { The University Of Texas At } \\
\text { Austin }\end{array}$ & Canvas, (Utilizada Como LMS), Blackboard Y Canvas \\
\hline 6 & Georgia Institute Of Technology & $\begin{array}{l}\text { WICHE Cooperative For Educational Technologies (WCET). WCET } \\
\text { Se Ha Asociado Con El Consorcio De Aprendizaje En Línea (OLC) Y } \\
\text { UPCEA }\end{array}$ \\
\hline 7 & $\begin{array}{l}\text { University Of California, Santa } \\
\text { Barbara }\end{array}$ & $\begin{array}{l}\text { Moodle2, Java- And HTML5- Actividades Interactivas Basadas, Y } \\
\text { Sesiones De Discusión Síncronas, Según Adobe Connectpro }\end{array}$ \\
\hline 8 & $\begin{array}{l}\text { The Imperial College Of } \\
\text { Science, Technology And } \\
\text { Medicine }\end{array}$ & Virtual Learning Environment (VLE) \\
\hline
\end{tabular}

Se aprecia que varias de estas instituciones tienen plataformas interconectadas y de apoyo tal como edX, implementada por el Instituto Tecnológico de Massachusetts y la Universidad de Harvard en 2012 con la finalidad de aportar a la investigación y el aprendizaje, siendo este un entorno virtual en donde se ofrecen cursos tanto para estudiantes como para personas particulares, además las plataformas internas poseen vastos repositorios, con la mejor tecnología, ya que estas se están actualizando constantemente, algunas poseen sistemas abiertos de gestión lo que permite la mejora continua. Varias de las tecnologías de otras universidades son muy utilizadas a nivel mundial tales como: Blackboard, Moodle, Canvas, etc. Muchos resultados de publicaciones, artículos indexados, proyectos tecnológicos, innovaciones, etc., son consecuencia del uso de estas plataformas virtuales gestionadas por los establecimientos educativos. De tal forma que, si se integran la acreditación o reconocimiento de una carrera con un reforzamiento basado en las TIC basadas en una gestión acertada, los resultados a los que conducen son sencillamente hacia la excelencia académica.

\section{Educación superior virtual actual}

La educación superior ha propuesto en los últimos años la formación profesional en pregrado y posgrado en una modalidad virtual. Varias Universidades han sido criticadas por los títulos expedidos, algunos casos muestran que no tienen ningún reconocimiento institucional, como contraparte existen varios ejemplos en los que existen títulos expedidos por universidades de reconocida trayectoria. Schneckenberg, muestra ejemplos de universidades que se basan totalmente en la formación virtual tales como: JIU (Jones International University), University of Phoenix, en Norteamérica, la UNED (Universidad Nacional de Educación a Distancia) en España, la Universidad a Distancia Hagen, en Alemania. (Schneckenberg, 2004, pág. 149) 
Silvio (2000), explica la forma que puede una institución ofrecer sus recursos de acceso a las tecnologías informativas para la educación y formación a través de tres tipos de interactividad.

$\checkmark$ Interactividad consultiva.- se refiere al uso y acceso del alumno a la información que existe en la base de datos de la universidad y bibliotecas a través de sistema informático (en la web) luego de que este haya realizado su proceso de matrícula.

$\checkmark$ Interactividad comunicacional.- aquí además de los beneficios anteriores el alumno puede tener una comunicación sincrónica (en chats, videoconferencias, etc.) o una comunicación asincrónica (mails, grupos de discusión, foros, etc.), además de tener comunicación con los demás usuarios (estudiantes del curso).

$\checkmark$ Interactividad transaccional.- es lo más recomendable en procesos de enseñanza, puesto que reúne las características de las anteriores interactividades y añade la gestión de parte y parte, tales como la supervisión de los alumnos generación de datos que realizan el seguimiento al alumnos, así como la gestión del propio alumno de su trabajo. Además cuenta con la ventaja de realizar procesos investigativos. (Silvio, 2000, págs. 199-200)

\section{La introducción y conducción de las TIC en la educación superior}

La actualización, la tecnología de punta, las herramientas web, interconexiones, y demás materiales, son ventajas que puede contener una Plataforma Virtual, pero el éxito de este sistema es su forma de gestión con la finalidad de tener eficiencia en la transferencia de conocimiento. De tal forma que se puede diferenciar claramente una Plataforma o una página web de una LMS (Sistema de Gestión de aprendizaje) en donde su principal virtud es su sistema de gestión.

El conocimiento puede referirse al proceso de transformación continua de datos en informaciones y luego estas informaciones en conocimientos y los conocimientos en nuevos productos, servicios o procesos (Canales, 2011), luego los nuevos conocimientos a través de la investigación vuelven a generar datos y nuevamente inicia dicho proceso.

Estos procesos deben ser planeados, conducidos y controlados continuamente, deben estar basados en objetivos claros en cada institución educativa, orientados a la solución de problemas, generación y modificación del conocimiento. Los problemas deben ser solucionados a través del conocimiento, de las bases de datos, de los repositorios, transferirlos a la realidad para modificarla y luego controlar los resultados obtenidos con los objetivos trazados. (Silvio, 2000, págs. 78-79)

El modelo clásico de organización de educación superior, se distingue por tres funciones básicas (Silvio, 2000, pág. 79): enseñanza, investigación y extensión.

La enseñanza se refiere a la interacción entre el alumno y su docente en donde el alumno recibe la información, la codifica y la transforma en su conocimiento, luego pasa a la función investigativa en donde se generan nuevos conocimientos y datos relevantes (por lo general 
producidos en la búsqueda de soluciones a problemas) los cuales deben ser transmitidos a la comunidad en general en el proceso de extensión.

Así, el proceso de gestión en la educación superior debe organizarse acorde a su naturaleza que es: enseñar, investigar, y extender el conocimiento; el sistema organizativo se regirá en los diferentes niveles: estratégico, táctico y operacional y el sistema de administrativo se basará en los niveles de: planeación, conducción y control; todo este sistema se puede acoplar como se muestra en la Figura 1, a pesar de que se pueda considerar un sistema complejo estas funciones se acoplan perfectamente, debe funcionar de forma dinámica y puede ser integrada los sistemas de gestión de aprendizaje en las plataformas virtuales.

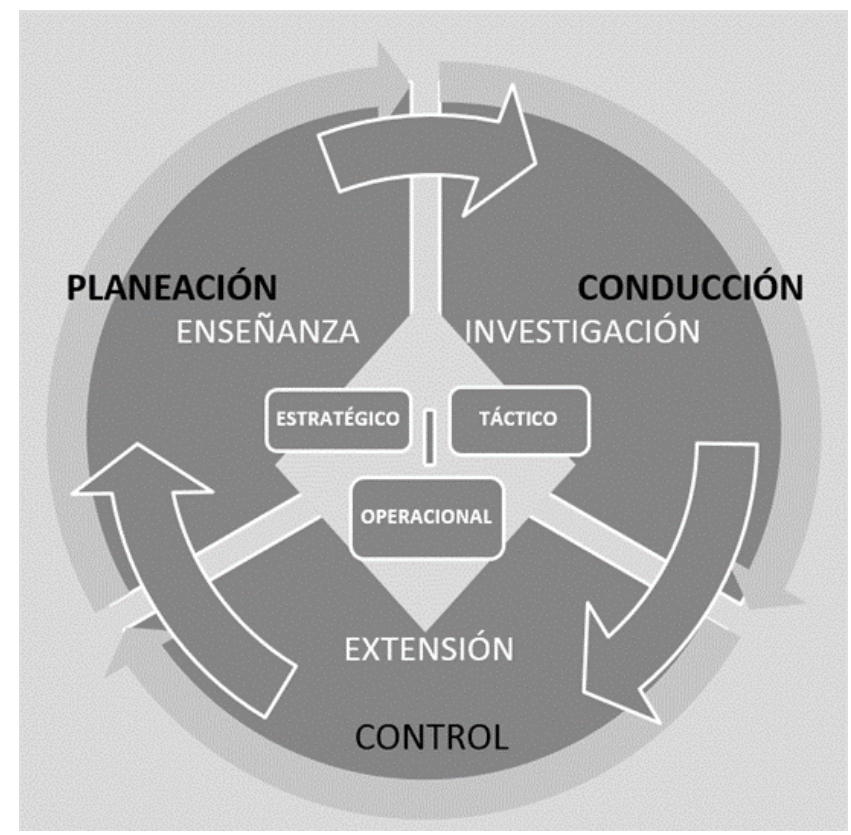

Figura 1. Proceso de gestión de la educación superior

\section{Fiabilidad y factores relevantes de una LMS}

Una plataforma debe otorgar varios beneficios como capacitación, facilidad de uso de las herramientas tecnológicas del internet, acceso a cualquier distancia y tiempo, ser de fácil uso y soporte, interacción entre alumnos y tutores, acceso a toda la información necesaria para lograr acceder a los diversos ritmos de aprendizaje. La utilización de una plataforma puede dar mejores resultados en su entorno si cuenta con ciertas particularidades como: alojamiento web, seguridad, corrección de errores, soporte pedagógico, soporte técnico, diseño gráfico, mantenimiento de servidor, mantenimiento de software, gestión de datos, actualización, instalación y configuración (Creative Commons, 2015).

Estos datos al ser agrupados nuevamente y tomando en cuenta el modelo organizativo de una universidad, se manifiestan los principales componentes de funcionamiento a los que comúnmente se alinea una plataforma gestionada para el aprendizaje como muestra el Gráfico 2; así el 45\% depende de la plataforma, de su presentación, su diseño, de su software, la actualización de sus herramientas el acceso del soporte que pueda brindar a sus usuarios. Luego 
con un 30\% está la calidad del servidor, en lo que se refiere a la seguridad de sus datos, a la capacidad de hosting, al mantenimiento y manejo de datos, por otra parte con un $20 \%$ se encuentra la gestión docente que pueda generar el profesor a través de la plataforma respecto a los datos que obtenga, al soporte que brinde, investigación y a la solución de problemas; por último el alumno presenta una gestión de un 5\%, esto refiriéndose a la configuración que pueda dar a la plataforma y el acceso al manejo de sus propios datos.

\section{Incidencia de Factores Relevantes en una Plataforma Virtual}

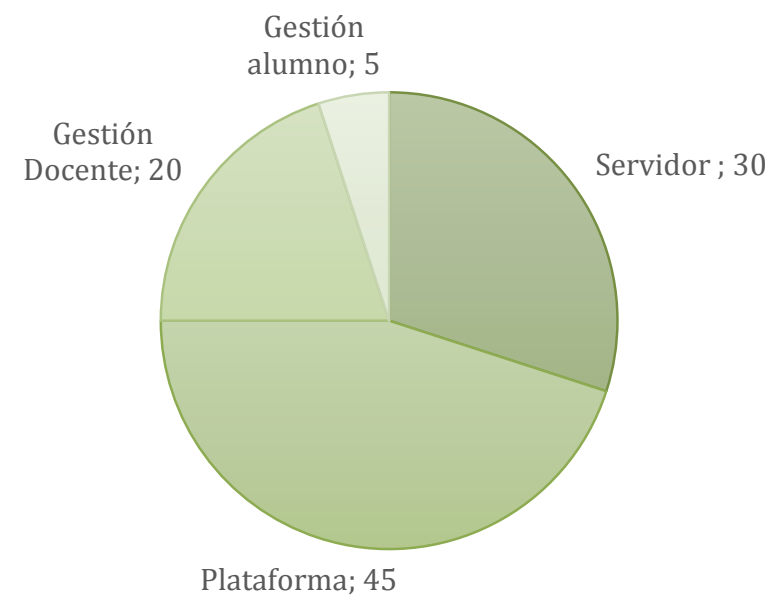

Grafico 2. Incidencia de factores relevantes en una plataforma virtual

\subsection{Criterios seguidos para la sección de plataforma virtual}

Macías (2010), en un estudio sobre el uso de plataformas de aprendizaje, concluye que la plataforma más usada es Moodle con más del 50\% en varias instituciones de educación superior. Esto puede ser por su accesibilidad, gratuidad y porque es una plataforma popular y conocida a todo nivel. De la misma manera muestra las calificaciones de la plataformas: Sakai, Claroline, Dokeos, y Moodle en donde los criterios seguidos para la selección de plataforma virtual fueron: Generalidades de la plataforma, Gestión y Administración, Sistemas de Comunicación, Gestión y producción de material didáctico y enseñanza, y Evaluación y Autoevaluación (Macías, 2010, págs. 73-74); estas plataformas fueron calificadas por expertos en donde se observa a través del grafico 3, que las mejores calificaciones las obtiene la plataforma Dokeos, la cual es una plataforma LMS con código abierto y que puede obtener colaboración interinstitucional en línea, por otro lado Moodle ofrece aulas virtuales basadas en el constructivismo y educación social. Cabe indicar que los resultados muestran una calificación de ciertas plataformas libres que son elitistas en las que Moodle está inmersa y por ende estos resultados son comparativos y dependen de la actualización y mejora que cada plataforma las haga internamente. Los criterios mostrados deben ser contrastados con las necesidades de la educación técnica superior para lograr la eficacia en el proceso de enseñanza-aprendizaje. 


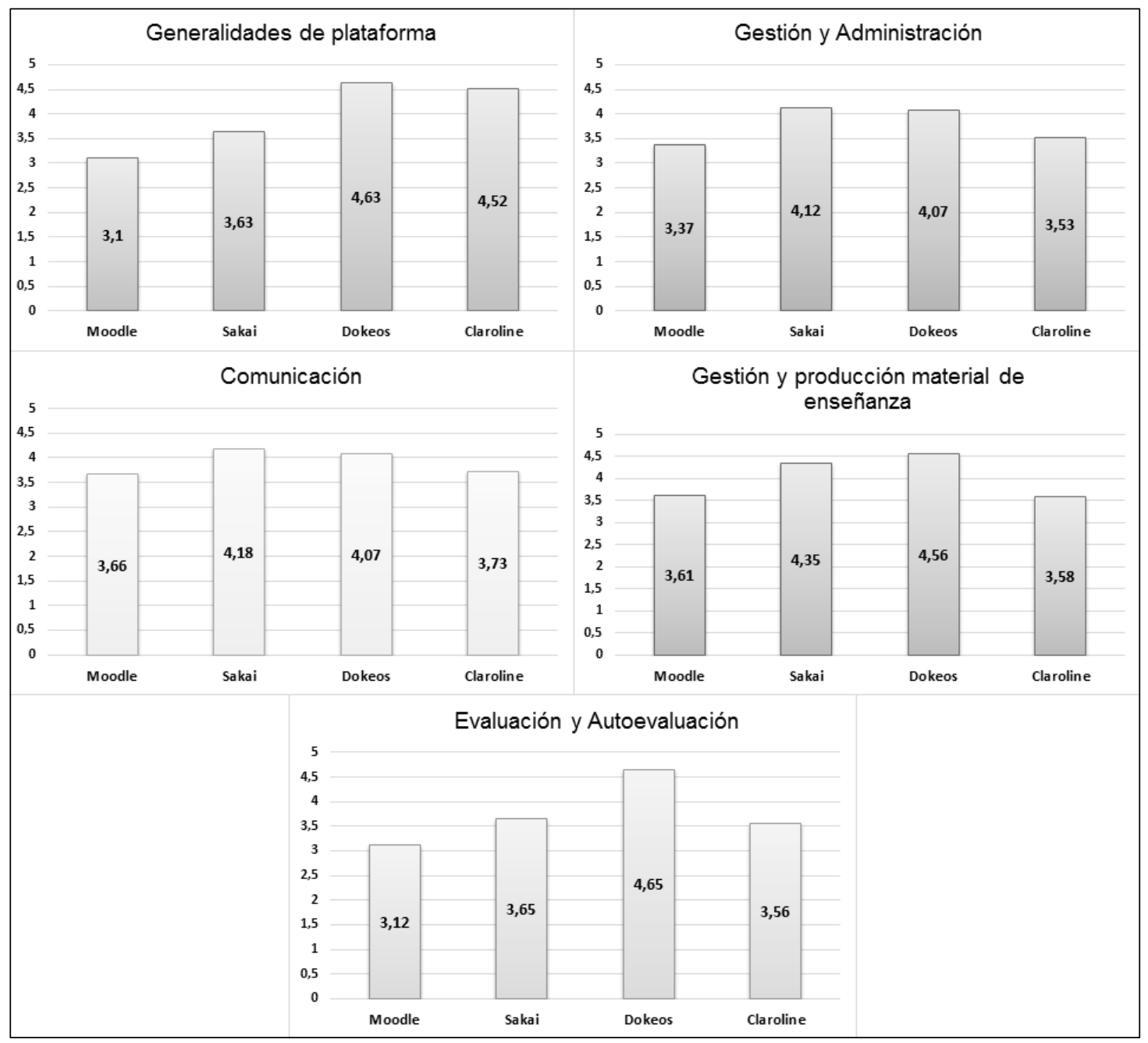

Grafico 3. Comparativa de plataformas virtuales

Existen varios investigadores que califican las plataformas virtuales acorde a las herramientas de aprendizaje, Zavahra (2012) por ejemplo, hace una clasificación de estas herramientas en criterios de Comunicación, Productividad y Participación estudiantil; estos son datos que se deben agrupar en conjunto con varias características complejas de gestión para poder obtener buenos resultados.

\section{Componentes de introducción de TIC in situ}

La introducción de las TIC dentro de las instalaciones institucionales depende varios factores internos que se deben analizar antes de elegir en introducirla una materia, en parte de un nivel o una carrera técnica en su totalidad. Ardila (2011), hace referencia a ciertos parámetros que se debe tener en cuenta al momento de adaptar una TIC en un sistema educativo los que pueden ser:

- Instalaciones y hardware, redes, conexiones

- Infraestructura lógica: software 
- Actores: personas que utilizan el hardware y el software para buscar, producir y administrar datos, informaciones y conocimientos.

- Recursos: datos, informaciones, conocimiento, bibliotecas electrónicas, grupos virtuales de investigación y discusión.

- Servicios telemáticos: de comunicación y búsqueda de información y navegación.

- Software de aplicaciones individuales. (Ardila, 2011, págs. 189-206)

Esto, en referencia a la introducción física de las TIC dentro del espacio físico de una institución de educación superior, esto se vuelve muy necesario puesto que muchas veces los estudiantes optan por utilizar las mismas instalaciones como su medio y espacio de trabajo.

Con respecto al software que utilizará una institución y su relación con la calidad, se debe tomar en cuenta la incidencia de factores relevantes y pensar que varios alumnos van a optar por tomar sus tareas y estudios como si se tratase de una modalidad a distancia, para esto, The Institute For Higher Education Policy (2000), realizó un estudio y elaboró una lista de puntos de referencia o parámetros que son esenciales para asegurar la calidad en la educación a distancia basada en Internet, esto con el fin de asegurar tanto la educación dentro del aula como fuera de ella. Estos parámetros pueden fundamentarse en: Soporte institucional, Enseñanza/Aprendizaje, estructura de la materia, apoyo estudiantil, apoyo a la carrera o facultad, evaluación y valoración. (The Institute For Higher Education Policy, 2000, págs. 2-3)

\subsection{Parámetros de soporte institucional}

La plataforma virtual debe contar con un sistema de seguridad electrónica, la cual asegure la calidad, integridad, validez y garantía de la información; la fiabilidad de software y la actualización de la tecnología deben ser elevadas incluso se debe probar el funcionamiento a prueba de fallos en lo posible. El sistema debe concentrar las sugerencias y proporcionar apoyo al rediseño, construcción y mantenimiento de la infraestructura de la educación virtual, de esta manera se pueden dar puntos de referencia para la construcción y reconstrucción de la infraestructura.

La institución debe estar clara respecto a la normativa interna del establecimiento y del desarrollo de cada materia, el uso e introducción de nuevas tecnologías debe estar restringida a los resultados de aprendizaje,; por ende también los materiales didácticos deben revisados periódicamente para garantizar que cumplan con las normas del programa, todos los cursos deben estar diseñados para que los estudiantes se comprometan con en el análisis, síntesis y evaluación dentro del proceso de aprendizaje.

\subsection{Parámetros de enseñanza/aprendizaje}

Se basa en la interacción del estudiante con los profesores, y también de la interacción del estudiante con otros estudiantes, esta característica esencial se facilita por medio de los correos electrónicos, chats, foros, y la tecnología para comunicación interna. Es de vital importancia la retroalimentación a las tareas y preguntas de los estudiantes, con la fundamental característica que la información debe llegar en el momento oportuno. Los estudiantes deben ser instruidos en 
la utilización de métodos apropiados de investigación efectiva, incluyendo la evaluación de la validez de los recursos que ellos están consultando para su aprendizaje.

\subsection{Parámetros de estructura de la materia}

Es recomendable que cada TIC esté asociada con los recursos necesarios para cada materia, y que cada estudiante se encuentre motivado y comprometido antes de iniciar el estudio de su materia, sin olvidar que el estudiante debe contar con recursos mínimos tecnológicos para su acceso.

Debe existir la información de los objetivos del curso, sus resultados de aprendizaje, los temas, en sí, debe informar como un syllabus completo. Cada materia debe contar con acceso a los repositorios para consultas y estudio del tema, en lo que se refiere a calificaciones, tiempos del curso, pruebas, inicio y finalización, porcentajes, etc., deben estar siempre visibles para que no existan dudas de los estudiantes.

\subsection{Parámetros de apoyo estudiantil}

Los estudiantes deben estar informados sobre los programas, incluyendo los requisitos de admisión, matrícula y cuotas, libros y suministros, requisitos técnicos y servicios de apoyo que brinda la institución. Además se debe capacitar para la obtención de material a través de bases de datos electrónicas, préstamos interbibliotecarios, archivos, servicios de noticias y otras fuentes. A lo largo del programa, deben tener acceso a asistencia técnica, las preguntas dirigidas al personal de asistencia de los estudiantes deben ser contestadas con precisión y rapidez.

\subsection{Parámetros de apoyo a la facultad}

Se debe prever asistencia técnica durante el desarrollo del curso para los profesores, además los miembros del profesorado pueden ser asistidos en la transición de la enseñanza en el aula para la enseñanza en línea y ser evaluados durante el proceso. Es recomendable que exista la capacitación al docente, incluido la tutoría entre pares, para esto el espacio debe contar con recursos escritos para solucionar las cuestiones originadas entre los estudiantes.

\subsection{Parámetros de evaluación y valoración}

Estos parámetros son útiles para el proceso de la eficacia en el proceso de enseñanza aprendizaje, para esto es necesario que el programa educativo se valide por medio de un sistema evaluativo, los métodos y normas aplicadas deben ser específicas y confiables. Los datos de matrícula, costos y usos exitosos e innovadores de la tecnología se utilizan para evaluar la efectividad del programa. (The Institute For Higher Education Policy, 2000, pág. 3)

Los resultados de aprendizaje previstos son revisados periódicamente (como sugerencia luego de cada cohorte o período semestral) para garantizar la claridad, utilidad y conveniencia respecto al medio que se desarrolle. 


\section{Conclusiones}

Las plataformas LMS conjuntamente con el docente, deben estar asociados entre sí, las primeras deben alinearse a las diversas teorías del aprendizaje y acorde a ellas introducir las herramientas necesarias para fines de enseñanza-aprendizaje; por otra parte el docente debe conocer lo anteriormente mencionado además de ser diestro en el manejo de tecnologías.

La educación técnica superior debe asumir el reto de acreditar sus carreras internacionalmente o en un espacio extenso, por ello las mejores universidades apuestan a la introducción de plataformas virtuales con fines de mejora en la eficacia de aprendizaje de sus alumnos, del acceso a la información, la movilidad y la investigación.

La organización educativa en las universidades debe basarse en los principios de enseñanza, investigación y extensión, todas ellas gestionadas en los procesos de planificación, conducción y control en sus diferentes niveles: estratégico, táctico y operacional.

La fiabilidad de los LMS están sujetos a las condiciones del servidor, las características y herramientas de la plataforma, la capacidad para la gestión docente, y la gestión del alumno, de la misma manera los resultados de los procesos educativos en estas plataformas se vinculan a los parámetros de educación virtual y a su constante evaluación de la eficacia educativa.

\section{Bibliografía}

Ardila, M. (2011). Indicadores de calidad de las plataformas educativas digitales. 14(1).

Canales, E. (2011). Gestión del Conocimiento en la Nueva Economía. Recuperado el 02 de Febrero de 2015, de http://unmsm-gestiondelconocimiento.blogspot.com/2012/07/gestion-delconocimiento.html

Center for World-Class Universities of Shanghai Jiao Tong University. (s.f.). Academic Ranking of World Universities. ( ) Recuperado el 12 de Enero de 2015, de http://www.shanghairanking.com/FieldENG2013.html

Creative Commons. (2015). Plataformas E-Learning, 3.0. (Creative Commons) Recuperado el 10 de Enero de 2015, de http://plataformas-e-learning.wikispaces.com/

EEES. (2015). Espacio Europeo de Educación Superior. Recuperado el 29 de Enero de 2015, de http://www.eees.es/es/eees

Macías, D. (2010). Plataformas de enseñanza virtual libres y sus características de extensión: Desarrollo de un bloque para la gestión de tutorías en Moodle. 1(1). Alcalá.

Paredes, J., \& Estebanell, M. (2005). Revista de educación: Convergencia Europea y Universidad. I (337). 
Salinas, J. (2004). La integración de las TIC en las instituciones de educación superior como proyectos de innovación educativa. I Congreso de Educación mediada con Tecnologías. Universidad de las Islas Baleares.

Sancho, J. (2002). Las Tecnologías De La Información Y La Comunicación En La Enseñanza Superior: Una Aproximación Compleja. Xiv (33).

Schneckenberg, D. (2004). El e-learning transforma la educación superior. I (33).

Silvio, J. (2000). La Virtualización De La Universidad: ¿Cómo transformar la educación superior con la tecnología? (Vol. I). Caracas: IESALC.

The Institute for Higher Education Policy. (2000). Benchmarks for Success in Internet-Based Distance Education. Washington: National Education Association.

Zavahra, Y. (2012). Plataformas Educativas. (Google Sites) Recuperado el 02 de Febrero de 2015, de https://sites.google.com/site/plataformaseducativasvirtuales/home/tipos/comparacion 